\title{
OHANA: Eta Carinae's Variability in the Near-IR
}

\section{Andrea Mehner, Willem-Jan de Wit, Thomas Rivinius and the Paranal VLTI group}

European Southern Observatory, Alonso de Cordova 3107, Vitacura, Chile email: amehner@eso.org

\begin{abstract}
Near-IR photometry of $\eta$ Car since 1972 revealed a long-term trend towards hotter temperatures and a cycloidal behavior of its near-IR colors around periastron passages. Both effects are likely triggered by the companion. We used VLTI AMBER observations from 20042014 to investigate $\eta$ Car's variabilities in the near-IR.
\end{abstract}

Keywords. stars: atmospheres, (stars:) circumstellar matter, stars: emission-line, Be, stars: individual (eta Carinae), stars: mass loss, stars: winds, outflows

\section{Introduction}

$\eta$ Car is one of the key objects for studying the evolution of the most massive stars. Two centuries ago it experienced an eruption in which the star expelled more than $10 M_{\odot}$, creating its bipolar nebula. The recent UV, optical, and X-ray light curves and spectra suggest a physical change in $\eta$ Car's wind over the last decade (Mehner et al. 2010, 2012). The near-IR photometry shows an accelerated brightening since 1998; the star became bluer, matching an apparent increase in blackbody temperature of an optically thick emitting plasma component from about $3500 \mathrm{~K}$ to $6000 \mathrm{~K}$ over the last 20 years (Mehner et al. 2014). This long-term color evolution occurs discontinuously at each periastron passage. In addition, a cycloidal behavior is observed in the colors from -40 to 100 days around periastron (Fig. 1). We investigated the spectral information from 2004-2014 VLTI AMBER data for changes in the near-IR emission lines to complement the recent results from $\eta$ Car's near-IR photometry.

\section{Eta Carinae's Near-IR Flux Spectrum}

From 2004-2014 spectro-interferometry of $\eta$ Car was carried out with VLTI AMBER, delivering both flux and visibility spectra simultaneously. $\eta$ Car's optically thick wind with an diameter on the order of 4.3 mas $(\approx 9 \mathrm{AU})$ in the K-band was resolved to be asymmetric (Weigelt et al. 2007). In early 2014, $\eta$ Car was observed with AMBER and several baselines as part of the OHANA project, a public Paranal/VLTI Observatory survey at High ANgular resolution of Active OB stars. The survey combines high spatial resolution (4-45 mas) with high spectral resolution $(\mathrm{R} \approx 12000)$ and covers the He I 2.059 and $\operatorname{Br} \gamma$ lines. Observations over several months with extensive $(\mathrm{u}, \mathrm{v})$-plane coverage allow to investigate temporal and spatial variations. Temporal variations in $\eta$ Car occur on a timescale of 2-3 weeks and strong small-scale structure can be observed at even the shortest baselines.

$\eta$ Car has two strong emission lines in the K band: He I 2.059 and Br $\gamma$. These contribute $\sim 3.5 \%$ to the $\mathrm{K}$ band flux at apastron. In the $\mathrm{H}$ band, the spectrum is dominated by the hydrogen Bracket series, which contribute $~ 8 \%$ of the total flux. During periastron 

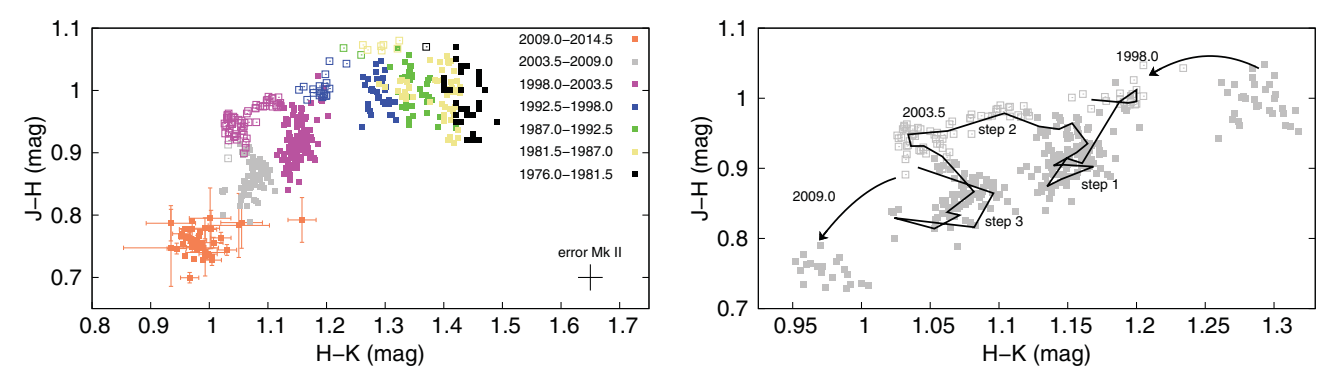

Figure 1. LEFT: Near-IR color-color diagram from 1976-2013. Open squares show data points close to periastron ( -40 days to +100 days). RIGHT: Cycloidal pattern for three orbital periods. Filled (open) squares are observations outside (during) periastron passages. The figures are adapted from Mehner et al. (2014). See also Whitelock et al. (2004) and references therein for details on the $\eta$ Car near-IR monitoring program at SAAO.

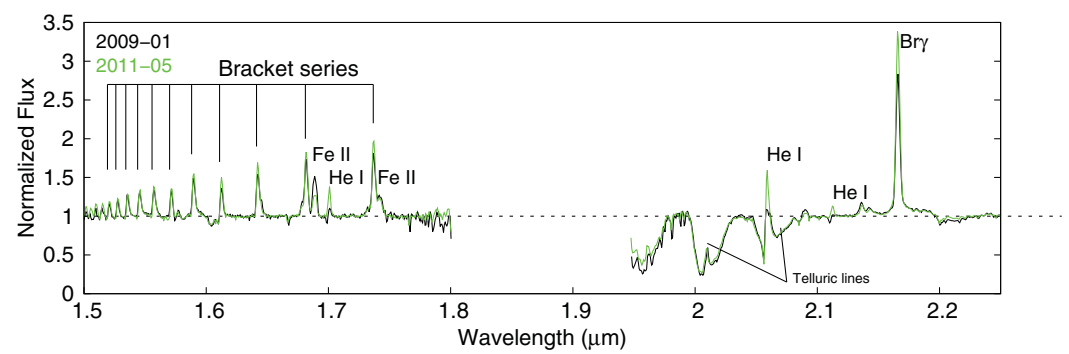

Figure 2. Medium-resolution H and K band VLTI AMBER spectra of $\eta$ Car during the 2009 periastron and the 2011 apastron when the influence of the companion star is minimal. The telluric bands are not corrected for.

passages the hydrogen lines weaken and only contribute $\sim 2 \%$ of the $\mathrm{K}$ band brightness and $\sim 4 \%$ of the $\mathrm{H}$ band brightness. These varying hydrogen line contributions are consistent with the $\mathrm{H}-\mathrm{K} \approx 0.1 \mathrm{mag}$ deviation in the color-color diagram observed close to periastron passages (Fig. 1, step 2). The $\mathrm{J}$ band is dominated by $\mathrm{Pa} \beta$, which contributes $\sim 10 \%$ of the total flux (Smith \& Davidson 2001), which is consistent with $\mathrm{J}-\mathrm{H} \approx$ constant during periastron passages.

\section{Conclusions}

We found no long-term evolution in the near-IR hydrogen and helium lines with respect to the continuum. The cycloidal behavior of the near-IR colors during periastron passages is accounted for by variations in the hydrogen emission line strengths with respect to the continuum. The long-term trend toward a hotter near-IR color is due to a change in the continuum and is triggered at periastron passages.

\section{References}

Mehner, A., Davidson, K., Humphreys, R. M., et al. 2012, ApJ 751, 73

Mehner, A., Davidson, K., Humphreys, R. M., et al. 2010, ApJ (Letters) 717, L22

Mehner, A., Ishibashi, K., Whitelock, P., et al. 2014, A\&SA 564, A14

Smith, N. \& Davidson, K. 2001, ApJ (Letters) 551, L101

Weigelt, G., Kraus, S., Driebe, T., et al. 2007, A\& A 464, 87

Whitelock, P. A., Feast, M. W., Marang, F., \& Breedt, E. 2004, MNRAS 352, 447 